\title{
Association between human glutathione S-transferase omega rs4925 polymorphism and bladder cancer
}

\author{
Su-Wei Hu ${ }^{1^{*}}$, Yen-Hao Su ${ }^{2 *}$, Zhon-Min Huang ${ }^{3 *}$, Hsin-An Chen ${ }^{2,4}$, Wei-Tang Kao ${ }^{1}$, \\ Yuan-Hung Wang ${ }^{4,5 \#}$, Chia-Chang $\mathrm{Wu}^{1 \#}$ \\ ${ }^{1}$ Department of Urology, Shuang Ho Hospital, Taipei Medical University, New Taipei City, Taiwan \\ ${ }^{2}$ Division of General Surgery, Department of Surgery, Shuang Ho Hospital, Taipei Medical University, Taipei, Taiwan \\ ${ }^{3}$ Department of Urology, Tung's Taichung MetroHarbor Hospital, Taichung County, Taiwan \\ ${ }^{4}$ Graduate Institute of Clinical Medicine, College of Medicine, Taipei Medical University, Taipei, Taiwan \\ ${ }_{5}^{5}$ Division of General Surgery, Department of Urology, Shuang Ho Hospital, Taipei Medical University, New Taipei City, Taiwan

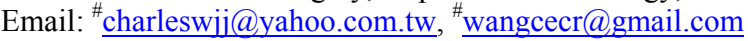

Received 26 October 2012; revised 27 November 2012; accepted 5 January 2013

\begin{abstract}
Glutathione S-transferases (GSTs) play an important role in the detoxification of polycyclic aromatic hydrocarbons and aromatic amines, the toxic substances contained in cigarettes. GST Omega 1 (GSTO1) not only utilizes glutathione in conjugation reaction but also contributes to the biotransformation of several xenobiotics. A single nucleotide polymorphism (Ala140Asp) of GSTO1 gene causing variations in enzyme activity may influence individual susceptibility to bladder cancer (BC). It is hypothesized that genetic polymorphism of GSTO1 gene has an effect on BC risk in particular by interacting with cigarette smoking. A total of histopathologically confirmed $300 \mathrm{BC}$ patients and 300 cancer-free controls were recruited from February 2002 to February 2009. Genotyping of the GSTO1 Ala140Asp polymorphism was determined using a polymerase chain reaction-restricted fragment length polymorphism (PCR-RFLP) method. The odds ratio (OR) and $95 \%$ confidence interval (CI) were calculated as a measure of the combined effect of cigarette smoking and the GSTO1 Ala140Asp polymorphism on BC risk. We found that study subjects with the GSTO1 Ala/Ala genotype have a significantly increased $B C$ risk $(O R=1.5 ; 95 \% C I=1.1$ 2.7). A statistically significant increased $B C$ risk was also found in ever smokers with the GSTO1 Ala/Ala genotype $(\mathrm{OR}=4.9 ; 95 \% \mathrm{CI}=2.8-9.7)$. In conclusion, this study provides an epidemiologic evidence of a significantly increased BC risk among ever smokers with the GSTO1 Ala/Ala genotype.
\end{abstract}

Keywords: Bladder Cancer; Cigarette Smoking; GSTO1;

\footnotetext{
*These authors contributed equally to this work.

${ }^{\#}$ Corresponding authors.
}

Polymorphism

\section{INTRODUCTION}

Bladder cancer is a multi-factorial malignancy influenced by environmental risk factors and molecular variations in metabolism-related genes leading to DNA instability and resulting in the development of cancer [1]. Cigarette smoking is a major risk factor for bladder cancer [2,3]. Cigarettes contain several carcinogens includeing polycyclic aromatic hydrocarbons, aromatic amines and $N$-nitroso compounds which have been reported to be associated with bladder cancer [4]. Epidemiological studies have indicated that cigarette smokers have a significantly higher bladder cancer risk $[3,5]$. Our previous study also found that cigarette smokers have a significantly increased risk than those who had never smoked [6]. Other risk factors including arsenic exposure through drinking well water, occupational exposure to carcinogens and chronic inflammation are known risk factors for bladder cancer $[7,8]$.

Carcinogens contained in cigarettes generally required the detoxification by phase II enzymes leading to lower toxic and more hydrophilic derivatives and then more readily excreted. However, the deficiency in detoxification-related enzymes generated oxidized products includeing reactive oxygen species (ROS) which can cause DNA damage and the accumulation of genetic mutations [9]. Several genetic polymorphisms of detoxificationrelated enzymes such as glutathione Stransferases (GSTs) which have been shown to be responsible for catalyzing several chemical carcinogens and may modulate the individual susceptibility to the development of various malignancies [10-12].

GST omega 1 (GSTO1) not only carried a cysteine at the active site but also had a specific N-terminal extension of $11-20$ amino acid residues [13]. GSTO1 also 
have thioltransferase and dehydroascorbate reductase activities [14]. In addition, GSTO1 can protect cells against calcium-induced apoptosis [15]. A single nucleotide polymorphism (rs4925) of GSTO1 gene was reported at nucleotide 419 causing an alanine (Ala) to aspartate (Asp) substitution in amino acid 140. This amino acid substitution of GSTO1 may lead to a lower enzyme activity of the variant enzyme in a substrate-specific manner [16]. In addition, the GSTO1 Asp/Asp gene genotype not only caused a substitution in amino acid from hydrophobic to hydrophilic residue but also exhibited the thiol-transferase activity only $75 \%$ of that of the Ala/Ala genotype [15-17]. Thus, certain genetic variations located in GSTO1 gene might explain the individual susceptibility to oxidative stress from cigarette smoking.

To explore the potential effect of the rs4925 polymorphism of GSTO1 gene on the risk of bladder cancer, we carried out a hospital-based case-control study to address this issue. Furthermore, we tested the joint effect of cigarette smoking and the rs4925 polymorphism of GSTO1 gene on the development of bladder cancer.

\section{MATERIALS AND METHODS}

\subsection{Study Population}

A total of 300 histopathologically confirmed bladder cancer $(\mathrm{BC})$ patients were enrolled from the Department of Urology, National Taiwan University Hospital between September 2002 and May 2009. The diagnosis was performed following the routine urological practices, including endoscopic biopsy or surgical resection of urinary tract tumors, with tissue samples confirmed by board-certified pathologists. In addition, a total of 300 cancer-free controls recruited from a hospital-based pool including those who received a general health examination at Taipei Medical University Hospital and Taipei Municipal Wan Fang Hospital. These controls were matched to BC patients in terms of age \pm 3 years, as well as gender, and had no history of malignancy. All participants provided informed consents and then were interviewed by a well-trained interviewer using a structured questionnaire to collect information including a history of cigarette smoking. Study subjects who had smoked more than 100 cigarettes during their lifetime were regarded as ever smokers, while those who had smoked less than 100 cigarettes were defined as never smokers. The number of pack-years was calculated using the formula: pack-years $=\left(\frac{\text { cigarettes perday }}{20}\right) \times($ smoked years). The Research Ethics Committee of the National Taiwan University Hospital, Taipei, Taiwan, approved this study, and this study also complied with the World Medical Association Declaration of Helsinki.

\subsection{Genotyping of GSTO1 Ala140Asp Polymorphism}

Genomic DNA was extracted from the buffy-coat of peripheral blood using standard methods and stored at $-80^{\circ} \mathrm{C}$. Genotyping was determined using a polymerase chain reaction-restriction fragment length polymorphism (PCR-RFLP) method. Briefly, the following primer sequences were designed for the GSTO1 Ala140Asp polymorphism: 5'-GAACTTGATGCACCCTTGGT-3' (forward) and 5'-TGATAGCTAGGAGAAATAAT TAC-3' (backward). The PCR conditions were: one cycle at $95^{\circ} \mathrm{C}$ for $5 \mathrm{~min}$; 35 cycles of $95^{\circ} \mathrm{C}$ for $30 \mathrm{sec}, 58^{\circ} \mathrm{C}$ for $30 \mathrm{sec}$ and $72^{\circ} \mathrm{C}$ for $45 \mathrm{sec}$, and a final extension at $72^{\circ} \mathrm{C}$ for 10 min. The amplified PCR product was 254 bp and visualized by electrophoresis in a $2 \%$ agarose gel. After the complete digestion with the restriction enzyme Cac8I at $37^{\circ} \mathrm{C}$ for 18 hours, the resulting DNA fragments which represented the Ala140Asp polymorphism of GSTO1 gene were analyzed by electrophoresis in a $3 \%$ agarose gels. To ensure quality, a random $10 \%$ of the samples were genotyped repeatedly.

\subsection{Statistical Analysis}

Tests of Hardy-Weinberg equilibrium (HWE) are performed using a $\chi^{2}$ goodness-of-fit test. The joint effects of cigarette smoking and the GSTO1 Ala140Asp polymorphism on $\mathrm{BC}$ risk were estimated by odds ratios (ORs) and its 95\% confidence intervals (CIs) using a multivariate-adjusted logistic regression. All statistical analyses were performed using the Statistical Analysis Software for Windows, version 9.1 (SAS Institute, Cary, NC). P-values of $<0.05$ were considered statistically significant.

\section{RESULTS}

\subsection{The Distribution of Basic Characteristics}

The distribution of basic characteristics for $\mathrm{BC}$ patients and controls is shown in Table 1. Among 300 BC patients, the mean age \pm SD (standard deviation) was $65.82 \pm 13.74$ years. Among 300 controls, the mean age \pm SD was $64.31 \pm 15.33$ years. There were no significant differences in the distribution of age, gender and education level between $\mathrm{BC}$ patients and controls. We found a significantly increased $\mathrm{BC}$ risk in ever smokers $(\mathrm{OR}=2.4 ; 95 \% \mathrm{CI}=1.5-3.9)$. The median of packyears among controls who had smoked was 30 packyears. Significantly increased BC risks of 2.1 and 2.4 were also found for study subjects who smoked $1-30$ pack-years and those who smoked more than 30 packyears, respectively. Moreover, we observed that alcohol drinkers had a significantly higher BC risk of $1.9(95 \%$ $\mathrm{CI}=1.3-3.4)$. 
Table 1. Demographic characteristics of $300 \mathrm{BC}$ patients and 300 controls.

\begin{tabular}{|c|c|c|c|}
\hline Variable & $\begin{array}{c}\text { BC patients } \\
\mathrm{n}(\%)\end{array}$ & $\begin{array}{c}\text { Controls } \\
\mathrm{n}(\%)\end{array}$ & OR $(95 \% \mathrm{CI})$ \\
\hline \multicolumn{4}{|l|}{ Age } \\
\hline$<50$ & $45(15.0)$ & $62(20.6)$ & 1.0 \\
\hline $50-59$ & $64(21.3)$ & $67(22.3)$ & $1.3(0.8-2.3)$ \\
\hline $60-69$ & $68(22.7)$ & $67(22.3)$ & $1.4(0.8-2.4)$ \\
\hline$\geq 70$ & $123(41.0)$ & $104(34.8)$ & $1.5(0.9-2.7)$ \\
\hline \multicolumn{4}{|l|}{ Gender } \\
\hline Female & $122(40.7)$ & $100(33.3)$ & 1.0 \\
\hline Male & $178(59.3)$ & $200(66.7)$ & $0.7(0.5-1.0)$ \\
\hline \multicolumn{4}{|l|}{ Education level } \\
\hline Illiteracy & $86(28.8)$ & $56(18.7)$ & 1.0 \\
\hline$\geq$ Elementary & $214(71.2)$ & $244(81.3)$ & $0.6(0.4-1.0)$ \\
\hline \multicolumn{4}{|c|}{ Cigarette smoking } \\
\hline Never & $157(52.3)$ & $193(64.3)$ & $1.0^{\mathrm{a}}$ \\
\hline Ever & $143(47.7)$ & $107(36.7)$ & $2.4(1.5-3.9)^{* * *}$ \\
\hline \multicolumn{4}{|c|}{ Cigarette smoking (pack-years) } \\
\hline 0 & $163(54.3)$ & $193(64.3)$ & $1.0^{\mathrm{a}}$ \\
\hline $1-30$ & $60(20.0)$ & $50(16.7)$ & $2.1(1.2-3.7)^{* *}$ \\
\hline$>30$ & $77(25.7)$ & $57(19.0)$ & $2.4(1.2-4.2)^{* *}$ \\
\hline \multicolumn{4}{|c|}{ Alcohol consumption } \\
\hline Never & $233(77.7)$ & $229(76.3)$ & $1.0^{\mathrm{b}}$ \\
\hline Ever & $67(22.3)$ & $71(23.7)$ & $1.9(1.3-3.4)^{*}$ \\
\hline
\end{tabular}

${ }^{*} \mathrm{P}<0.05,{ }^{* *} \mathrm{P}<0.01,{ }^{* * *} \mathrm{P}<0.001 ;{ }^{\text {a Adjusted for age, gender, education level and alcohol consumpti; }{ }^{\mathrm{b}} \text { Adjusted for age, gender, education leveland cigarette }}$ smoking.

\subsection{Comparison of the GSTO1 Ala140Asp Polymorphism}

The distribution of the observed genotype frequencies among the control group was in HWE for the GSTO1 Ala140Asp polymorphism $(\mathrm{P}=0.528)$. Study subjects who carried the Asp/Asp genotype of GSTO1 gene had a non-significant higher $\mathrm{BC}$ risk $(\mathrm{OR}=1.1 ; 95 \% \mathrm{CI}=0.4$ 4.6) comparing to individuals who carried the Ala/Ala genotype. However, compared with study subjects who carried the combination of Asp/Asp and Ala/Asp genotypes of GSTO1 gene, those with the Ala/Ala genotype had a significantly higher BC risk of $1.5(95 \%$ CI $=1.1$ - 2.7) (Table 2).

\subsection{Combined Effects of Smoking and the GSTO1 Ala140Asp Polymorphism}

Because we hypothesized that single nucleotide poly- morphisms located in GSTO1 gene would modulate the toxic effect of chemical carcinogens in cigarettes on $\mathrm{BC}$, we further examined the combined effect of the GSTO1 Ala140Asp polymorphism and cigarette smoking. Comparing with never smokers who carried Asp/Asp and Ala/Asp genotypes of the GSTO1 gene as the reference group, significantly increased $\mathrm{BC}$ risks of $1.9(95 \% \mathrm{CI}=$ $1.1-3.1), 3.1(95 \% \mathrm{CI}=1.5-6.5)$ and $4.9(95 \% \mathrm{CI}=2.8$ - 9.7) were found for never smokers carrying the Ala/Ala genotype, ever smokers with Asp/Asp and Ala/Asp genotypes and ever smokers with the Ala/Ala genotype, respectively (Table 3 ).

\section{DISCUSSION}

Chronic exposure to chemical carcinogens from cigarette smoking can lead to DNA damage adducts [9]. In consistent with previous studies, significantly increased BC 
Table 2. Distribution of GSTO1 Ala140Asp polymorphism in $300 \mathrm{BC}$ patients and 300 controls.

\begin{tabular}{cccc}
\hline Polymorphism & $\begin{array}{c}\text { BC patients } \\
\mathrm{n}(\%)\end{array}$ & $\begin{array}{c}\text { Controls } \\
\mathrm{n}(\%)\end{array}$ & $\mathrm{OR}^{\mathrm{a}}(95 \% \mathrm{CI})$ \\
\hline GSTO1 & & & 1.0 \\
Ala/Ala & $230(76.7)$ & $196(65.3)$ & $0.5(0.4-1.0)$ \\
Ala/Asp & $63(21.0)$ & $98(32.7)$ & $1.1(0.4-4.6)$ \\
Asp/Asp & $7(2.3)$ & $6(2.0)$ & 1.0 \\
Asp/Asp +Ala/Asp & $70(23.3)$ & $104(34.7)$ & $1.5(1.1-2.7)^{* *}$ \\
Ala/Ala & $230(76.7)$ & $196(65.3)$ & \\
\hline
\end{tabular}

${ }^{* *} \mathrm{P}<0.01 ;{ }^{a}$ Adjusted for age, gender, education level, cigarette smoking and alcohol consumption.

Table 3. Combined effect of GSTO1 Ala140Asp polymorphism and cigarette smoking on BC risk.

\begin{tabular}{ccccc}
\hline $\begin{array}{c}\text { Cigarette } \\
\text { smoking }\end{array}$ & GSTO1 genotype & $\begin{array}{c}\text { BC patients } \\
\mathrm{n}(\%)\end{array}$ & $\begin{array}{c}\text { Controls } \\
\mathrm{n}(\%)\end{array}$ & $\mathrm{OR}^{\mathrm{b}}(95 \% \mathrm{CI})$ \\
\hline \multirow{2}{*}{ Never } & Asp/Asp + Ala/Asp & $30(10.0)$ & $81(27.0)$ & 1.0 \\
& Ala/Ala & $100(33.3)$ & $120(40.0)$ & $1.9(1.1-3.1)^{*}$ \\
\multirow{2}{*}{ Ever } & Asp/Asp + Ala/Asp & $40(13.3)$ & $23(7.7)$ & $3.1(1.5-6.5)^{* *}$ \\
& Ala/Ala & $130(43.4)$ & $76(25.3)$ & $4.9(2.8-9.7)^{* * *}$ \\
& & & & $P_{\text {trend }}<0.0001$ \\
\hline
\end{tabular}

${ }^{*} \mathrm{P}<0.05 ;{ }^{* *} \mathrm{P}<0.01 ;{ }^{* * *} \mathrm{P}<0.001 ;{ }^{\mathrm{b}}$ Adjusted for age, gender, education level and alcohol consumption.

risks of $2.4,2.1$ and 2.4 were found for ever smokers, light smokers (1 - 30 pack-years) and heavy smokers (>30 pack-years), respectively. In addition, we observed a significantly increased UC risk of 1.9 for alcohol drinkers. Some studies reported that cigarette smoking and alcohol consumption have synergistic interaction on tumorigenesis [18].

GSTO1 has been found in various human tissues [15]. We observed that study subjects who carried the Asp/Asp genotype of the GSTO1 Ala140Asp polymorphism had a slightly higher BC risk. A previous study reported that significantly increased risks of hepatocellular carcinoma, cholangiocarcinoma and breast cancer were found for those carrying the Asp allele of the GSTO1 Ala140Asp polymorphism [19]. A study reported that the enzyme activity of GSTO1 Ala/Asp genotype was $75 \%$ of that of GSTO1 Ala/Ala genotype indicating this genetic variation may lead to defective protection against oxidative stresses [15]. However, several studies reported that the GSTO1 Ala140Asp polymorphism would not influence the thioltransferase activity and further modify the risk of malignancy [20-23]. The discrepancies between our findings and previous studies may be due to ethnic variation. The Asp allele frequency was significantly different among various populations. The Asp allele frequency was 0.34 for European population, 0.08 for Africans, 0.17 for Han Chinese and 0.12 for Japanese [19]. Our findings showed an Asp allele frequency of 0.18 which was similar to Asian population such as Han Chinese and Japanese.

The present study also found that a significant joint effect of cigarette smoking and the Ala/Ala genotype of the GSTO1 Ala140Asp polymorphism on BC risk (OR = 4.9), showing a dose-response relationship ( $P_{\text {trend }}<$ 0.0001). Therefore, our results implied that several environmental risk factors should be diminished to prevent individuals from the development of BC. This study has some limitations. Other factors including secondhand smoke exposure may modify the risk of $\mathrm{BC}$; however, lack of detailed information on exposure of environmental tobacco smoke in this study. Moreover, we select only one functional SNP of GSTO1 gene which might not exhibit entire expression and enzyme activity of GSTO1 [24,25]. Therefore, more functional SNPs of GSTO1 gene and other candidate genes should be included in a future study with a larger sample size.

In conclusion, we carried out a hospital-based casecontrol study to investigate the joint effect of cigarette smoking and the GSTO1 Ala140Asp polymorphism (rs4925) on the development of bladder cancer. Our findings showed that a significantly highest risk of bladder cancer was found in subjects who had smoked and carried the Ala/Ala genotype of GSTO1 gene. Therefore, we suggest that the decreasing of environmental risk factors could prevent individuals from the risk of bladder 
cancer, especially for those who carried the risk genotypes of detoxification-related genes.

\section{REFERENCES}

[1] Volanis, D., Kadiyska, T., Galanis, A., Delakas, D., Logotheti, S. and Zoumpourlis, V. (2010) Environmental factors and genetic susceptibility promote urinary bladder cancer. Toxicology Letters, 193, 131-137.

doi:10.1016/j.toxlet.2009.12.018

[2] Zeegers, M.P., Goldbohm, R.A. and van den Brandt, P.A. (2002) A prospective study on active and environmental tobacco smoking and bladder cancer risk (The Netherlands). Cancer Causes and Control, 13, 83-90. doi:10.1023/A:1013954932343

[3] Samanic, C., et al. (2006) Smoking and bladder cancer in Spain: Effects of tobacco type, timing, environmental tobacco smoke, and gender. Cancer Epidemiology, Biomarkers \& Prevention, 15, 1348-1354. doi:10.1158/1055-9965.EPI-06-0021

[4] International Agency for Research on Cancer (1986) IARC monographs on the evaluation of the carcinogenic risk of chemicals to humans: Tobacco smoking. World Health Organization, Lyon.

[5] Strope, S.A. and Montie, J.E. (2008) The causal role of cigarette smoking in bladder cancer initiation and progression, and the role of urologists in smoking cessation. Journal of Urology, 180, 31-37. doi:10.1016/j.juro.2008.03.045

[6] Wu, C.C., et al. (2012) Joint effect of arsenic methylation profile and NNK metabolites on urothelial carcinoma. Journal of Urology, 188, 1701-1705. doi:10.1016/j.juro.2012.07.025

[7] Kalble, T. (2001) Etiopathology, risk factors, environmental influences and epidemiology of bladder cancer. Urologe A, 40, 447-450.

[8] Pelucchi, C., Bosetti, C., Negri, E., Malvezzi, M. and La Vecchia, C. (2006) Mechanisms of disease: The epidemicology of bladder cancer. Nature Clinical Practice Urology, 3, 327-340. doi:10.1038/ncpuro0510

[9] Vineis, P. and Porta, M. (1996) Causal thinking, biomarkers, and mechanisms of carcinogenesis. Journal of Clinical Epidemiology, 49, 951-956. doi:10.1016/0895-4356(96)00118-7

[10] Di Pietro, G., Magno, L.A. and Rios-Santos, F. (2010) Glutathione S-transferases: An overview in cancer research. Expert Opinion on Drug Metabolism \& Toxicology, 6, 153-170. doi:10.1517/17425250903427980

[11] Simic, T., Savic-Radojevic, A., Pljesa-Ercegovac, M., Matic, M. and Mimic-Oka, J. (2009) Glutathione Stransferases in kidney and urinary bladder tumors. Nature Reviews Urology, 6, 281-289. doi:10.1038/nrurol.2009.49

[12] Landi, S. (2000) Mammalian class theta GST and differential susceptibility to carcinogens: A review. Mutation Research, 463, 247-283. doi:10.1016/S1383-5742(00)00050-8

[13] Whitbread, A.K., et al. (2005) Characterization of the omega class of glutathione transferases. Methods in Enzymology, 401, 78-99.

\section{doi:10.1016/S0076-6879(05)01005-0}

[14] Schmuck, E.M., et al. (2005) Characterization of the monomethylarsonate reductase and dehydroascorbate reductase activities of Omega class glutathione transferase variants: Implications for arsenic metabolism and the age-at-onset of Alzheimer's and Parkinson's diseases. Pharmacogenetics and Genomics, 15, 493-501. doi:10.1097/01.fpc.0000165725.81559.e3

[15] Tanaka-Kagawa, T., et al. (2003) Functional characterization of two variant human GSTO 1-1s (Ala140Asp and Thr217Asn). Biochemical and Biophysical Research Communications, 301, 516-520. doi:10.1016/S0006-291X(02)03066-8

[16] Whitbread, A.K., Tetlow, N., Eyre, H.J., Sutherland, G.R. and Board, P.G. (2003) Characterization of the human Omega class glutathione transferase genes and associated polymorphisms. Pharmacogenetics, 13, 131-144. doi:10.1097/01.fpc.0000054062.98065.6e

[17] Xu, Y., et al. (2009) Lack of association of glutathione-Stransferase omega 1 (A140D) and omega 2 (N142D) gene polymorphisms with urinary arsenic profile and oxidative stress status in arsenic-exposed population. Mutation Research, 679, 44-49. doi:10.1016/j.mrgentox.2009.07.008

[18] Schlecht, N.F., et al. (1999) Interaction between tobacco and alcohol consumption and the risk of cancers of the upper aero-digestive tract in Brazil. American Journal of Epidemiology, 150, 1129-1137.

[19] Marahatta, S.B., et al. (2006) Polymorphism of glutathione S-transferase omega gene and risk of cancer. Cancer Letters, 236, 276-281. doi:10.1016/j.canlet.2005.05.020

[20] Granja, F., Morari, E.C., Assumpção, L.V. and Ward, L.S. (2005) GSTO polymorphism analysis in thyroid nodules suggest that GSTO1 variants do not influence the risk for malignancy. European Journal of Cancer Prevention, 14, 277-280. doi:10.1097/00008469-200506000-00013

[21] Board, P.G. (2011) The omega-class glutathione transferases: Structure, function, and genetics. Drug Metabolism Reviews, 43, 226-235. doi: $10.3109 / 03602532.2011 .561353$

[22] Mukherjee, B., et al. (2006) Glutathione S-transferase omega 1 and omega 2 pharmacogenomics. Drug Metabolism and Disposition, 34, 1237-1246. doi:10.1124/dmd.106.009613

[23] Board, P.G. and Anders, M.W. (2007) Glutathione transferase omega 1 catalyzes the reduction of S-(phenacyl) glutathiones to acetophenones. Chemical Research in Toxicology, 20, 149-154. doi:10.1021/tx600305y

[24] Sanguansin, S., Petmitr, S., O-Charoenrat, P. and Pongstaporn, W. (2012) Association of glutathione S-transferase omega gene polymorphisms with progression of head and neck cancer. Molecular Biology Reports, 39, 10915-10920. doi:10.1007/s11033-012-1991-3

[25] Yanbaeva, D.G., Wouters, E.F., Dentener, M.A., Spruit, M.A. and Reynaert, N.L. (2009) Association of glutathione-S-transferase omega haplotypes with susceptibility to chronic obstructive pulmonary disease. Free Radical Research, 43, 738-743. doi:10.1080/10715760903038440 